\title{
Serum Biochemistry of Broiler Chicken Supplemented with Detoxifying Microbial Enzymes to Ameliorate Feed Toxins
}

\author{
S. Senthilkumar* , R. Kavitha, S.R. Janani, K.P. Prabhakaran, P. Vasanthakumar, \\ M.R. Purushothaman and D. Chandrasekaran
}

Department of Animal Nutrition, Veterinary College and Research Institute, (Tamil Nadu Veterinary and Animal Sciences University) Namakkal - 637002, Tamil Nadu, India

*Corresponding author

\section{A B S T R A C T}

\section{Keywords}

Broiler, Detoxifying microbial enzymes, Serum biochemistry

Article Info

Accepted:

17 June 2019

Available Online:

10 July 2019
A feeding trial was conducted to assess the efficacy of serum profile of broiler chicken supplemented with detoxifying microbial enzymes (DME) on feed toxins using 420 number of day -old male Vencobb broiler chicks were divided into fourteen groups, 30 birds in each for 42 days. Treatment groups: $\mathrm{T}_{1}$ and $\mathrm{T}_{2}$ were healthy control and positive control, respectively, $\mathrm{T}_{3}$ received naturally mycotoxin contaminated feed at field level, $\mathrm{T}_{4}$ received naturally mycotoxin contaminated feed at field level + DME at 250g/ton of feed, $\mathrm{T}_{5}$ received naturally contaminated feed with Clostridium spp., $\mathrm{T}_{6}$ received naturally contaminated feed with Clostridium spp.,+ DME at $250 \mathrm{~g} /$ ton of feed, $\mathrm{T}_{7}$ received naturally contaminated feed with E.coli, $\mathrm{T}_{8}$ received naturally contaminated feed with E.coli + DME at $250 \mathrm{~g} /$ ton of feed, $\mathrm{T}_{9}$ infected with Clostridium spp., on day $8^{\text {th }}, \mathrm{T}_{10}$ infected with Clostridium spp., on day $8^{\text {th }}+\mathrm{DME}$ at $250 \mathrm{~g} / \mathrm{ton}$ of feed, $\mathrm{T}_{11}$ infected with E.coli on day $8^{\text {th }}, \mathrm{T}_{12}$ infected with E.coli on day $8^{\text {th }}+$ DME at $250 \mathrm{~g} /$ ton of feed, $\mathrm{T}_{13}$ received mycotoxin contaminated feed inoculated with Clostridium spp., and E.coli from day $8^{\text {th }}$ and $\mathrm{T}_{14}$ received mycotoxin contaminated feed inoculated Clostridium spp., and E.coli from day $8^{\text {th }}+\mathrm{DME}$ at $250 \mathrm{~g} /$ ton of feed. At the end of $28^{\text {th }}$ and $42^{\text {nd }}$ day two birds from each replicate were slaughtered for biochemical analysis. The level of serum sodium, potassium, calcium, chloride, glucose, total protein, uric acid, SGOT, SGPT and ALP were measured. All the parameter levels were comparable with the treatment groups and it could be inferred that the supplementation of DME did not cause any adverse health effect on broilers.

\section{Introduction}

Mycotoxins are the toxic metabolites produced by certain fungi, mainly by the Aspergillus, Penicillium and Fusarium genera. They are always a hazard to man and domestic animals and had come to public interest since the past 30 years. Aflatoxins constitute a great threat to the health of animals and humans due to their teratogenic, carcinogenic, mutagenic and immunosuppressive effects (Guan et al., 2008; Yunus et al., 2011). Additionally, in terms of the livestock industry aflatoxins cause huge economic loss by retarding animal growth, increasing feed consumption and reducing meat production (Fan et al., 2013; 
Do and Choi, 2007). Due to its ubiquitous presence and harmful effect on consumption of contaminated feed, animal nutritionists focused their research to minimize the incidence of fungi in feed and toxicity from the toxin variety. In addition to fungal toxins, the poultry is also being exposed to bacterial toxins like Clostridium spp., and E.coli. Both these fungal and bacterial toxins known to affect the vital organs like liver, kidney and gut health in poultry. Since the vital organs and gut health are affected, the absorption and utilization of nutrients will be impaired which results in sub-optimum productive performance in broilers. The Food and Agriculture Organization (FAO) estimates that at least $25 \%$ of world cereal production is contaminated with mycotoxins (Dowling, 1997). Hence the research was carried out with detoxifying microbial enzymes (DME) which are capable of detoxifying the fungal and bacterial toxins and also contain toxin absorbents like MOS (Mannan Oligo saccharides), antifungal agents and biological antioxidants. Among mycotoxin binder, the use of biological methods, using microorganisms and their metabolites to eliminate aflatoxins can be a highly promising approach owing to its specific, efficient and environmentally sound detoxification (FAO, 2001). The present study was designed to ascertain the efficacy of detoxifying effect of the DME in broiler feed and its effects on serum biochemical parameters in broilers.

\section{Materials and Methods}

A biological trail was carried out with detoxifying microbial enzymes in broiler ration at Department of Animal Nutrition, Veterinary College and Research Institute, Namakkal with 420 numbers of day-old Vencobb straight run male chicks. The design of experiment followed was completely randomised design. The chicks were randomly divided in to fourteen groups. The experiment was conducted in a shed having Mangalore tile roofing and concrete flooring. The birds were housed in deep litter pens using coconut coir pith as litter material and reared from dayold to 6 weeks following standard management practices. Feed and water were provided ad-libitum. All the birds were vaccinated against Ranikhet disease on $7^{\text {th }}$ day and IBD (Infectious bursal disease) on $14^{\text {th }}$ day of age. Mortality was recorded on occurrence. Post-mortem was done and the cause of death was recorded.

\section{Experimental design and feeding program}

The chicks were weighed individually, wing banded and distributed randomly to fourteen treatment groups with three replicates with ten birds in each replicate. The broiler pre-starter, starter and finisher rations were fed from 0-14, 15-28 and 29-42 days of age, respectively. The treatment of the biological experiment was presented in Table 1.

The broiler pre-starter, starter and finisher rations were fed from 0-14, 15-28 and 29-42 days of age, respectively. The experimental rations were formulated to contain the same level of protein; energy, lysine, methionine, calcium and phosphorus by using MS excel ${ }^{(\mathrm{R})}$. The ingredient and proximate composition of the experimental prestarter, starter and finisher rations are presented in Table 2.

\section{Serum Preparation}

At the end of experiment, two birds from each replicate were slaughtered at the end of $28^{\text {th }}$ and $42^{\text {nd }}$ day for biochemical studies. About 10-15 ml blood was collected from each bird in a sterile test tube without any anticoagulant kept in a slanting position at room temperature for preparation of serum. These serum samples were refrigerated overnight at $4^{\circ} \mathrm{C}$. The separation of serum from the clotted blood was done by centrifugation at $1000 \mathrm{rpm}$ 
for $10 \mathrm{~min}$. These cell free serum samples were preserved at $-20^{\circ} \mathrm{C}$ for further biochemical analysis.

\section{Biochemical Parameters}

The biochemical parameters were analyzed with the preserved serum samples. The levels of sodium, potassium and calcium in serum were quantified using flame photometer as per AOAC (2002). The chloride content in feed was determined by thiocyanate method using diagnostic kit supplied by Span Diagnostics Ltd, India. Serum glucose (GOD/POD method), uric acid (kit method), total protein (Biuret method), serum glutamate oxaloacetate transaminase (SGOT) (IFCC method), SGPT (kit method) and alkaline phosphatase (ALP) (using alkaline phosphatase diagnostic kit method) levels were also determined.

\section{Statistical Analysis}

The data were collected and subjected to one way ANOVA using SPSS software as per the standard statistical methods given by Snedecor and Cochran (1994).

\section{Results and Discussion}

The data on serum profile of broilers fed with DME in different treatment groups during starter and finisher phase are presented in Table 3, 4 and 5, respectively. Sodium $(\mathrm{mEq} / \mathrm{L})$ level was significantly differed $(\mathrm{P} \leq 0.05)$ between the treatment groups in starter and finisher phase. In starter phase, it was lower in $\mathrm{T}_{7}(50.40 \pm 1.68)$ and higher in $\mathrm{T}_{2}$ group (159.64 \pm 5.91$)$. In finisher phase, lower levels were observed in $\mathrm{T}_{7}(121.87 \pm 4.09)$ and higher in $\mathrm{T}_{3}(164.71 \pm 6.99)$ when compared to other treatment groups. However, higher levels in both starter and finisher phase were comparable with control. Potassium $(\mathrm{mEq} / \mathrm{L})$ level in starter and finisher phase did not vary significantly but numerically vary among the treatment groups. In starter phase, lower level was found in $\mathrm{T}_{11}(4.90 \pm 1.55)$ and higher in $\mathrm{T}_{14}$ (20.19 \pm 9.80$)$. In finisher phase, potassium level was lower in $\mathrm{T}_{9}(8.26 \pm 1.34)$ and higher in $\mathrm{T}_{4}$ group (13.07 \pm 4.23$)$.

Calcium $(\mathrm{mEq} / \mathrm{L})$ level in starter phase significantly differ among the groups lower level was found in $\mathrm{T}_{3}(8.43 \pm 0.93)$ and higher level was observed in $\mathrm{T}_{6}(9.50 \pm 0.25)$. In finisher phase, the calcium level did not vary among the treatment groups and comparable with the control. Chloride $(\mathrm{mEq} / \mathrm{L})$ (starter and finisher) levels were comparable and not affected by the supplementation of DME. Uric acid levels $(\mathrm{mcg} / \mathrm{dl})$ were comparable and significantly vary among the treatment groups in starter and finisher phase. DME supplementation did not alter the serum glucose $(\mathrm{mg} / \mathrm{dl})$, protein $(\mathrm{g} \%)$ and liver enzymes such as SGOT (U/L), SGPT(U/L) and ALP (U/L) levels in starter and finisher phase.

El-Katcha et al., (2017) reported that the amount of calcium increased in the group treated with biological toxin binders when compared to other groups. Mycotoxin binder supplement significantly decreased the levels of serum urea, uric acid, but increased the liver related enzymes including the GOT and GPT levels when compared to control. However, some reports reported that mycotoxin binder increased serum ALP and ALT in animals (Bagherzadeh Kasmani et al., 2012). Aflatoxin toxicity in broiler may manifested by increased hepatic enzyme activities such as AST and ALT; and if AST and ALP are found together in elevated amount of blood and liver damage (Nyirenda and Makhambera, 1986). Aflatoxin increase lipid peroxidation in liver and kidney tissues and induce cellular damage causing impaired morphology of the organs (Verma and Chakraborty, 2008; Darwish et al., 2011). 
Table.1 Dietary treatment for the biological experiment

\begin{tabular}{|c|c|c|}
\hline $\begin{array}{l}\text { Treatment } \\
\text { Group }\end{array}$ & Purpose & Particulars and Dosage \\
\hline $\mathbf{T}_{1}$ & Healthy Control & Non - contaminated and non - medicated \\
\hline $\mathbf{T}_{2}$ & Positive Control & $\begin{array}{l}\text { Non - contaminated and non - medicated + DME @ } 250 \mathrm{~g} / \\
\text { ton feed. }\end{array}$ \\
\hline $\mathbf{T}_{3}$ & $\begin{array}{l}\text { Natural } \\
\text { Contaminant* }\end{array}$ & Naturally mycotoxin contaminated feed at field level. \\
\hline $\mathbf{T}_{4}$ & Prophylaxis* & $\begin{array}{l}\text { Naturally mycotoxin contaminated feed at field level }+ \\
\text { DME @ } 250 \mathrm{~g} / \text { ton feed. }\end{array}$ \\
\hline $\mathbf{T}_{5}$ & Infected Control & Naturally contaminated feed with Clostridium spp., \\
\hline $\mathbf{T}_{6}$ & Prophylaxis & $\begin{array}{l}\text { Naturally contaminated feed with Clostridium spp., + } \\
\text { Supplementation of DME @ } 250 \mathrm{~g} / \text { ton feed. }\end{array}$ \\
\hline $\mathbf{T}_{7}$ & Infected Control & Naturally contaminated feed with E.coli. \\
\hline $\mathbf{T}_{8}$ & Prophylaxis & $\begin{array}{l}\text { Naturally contaminated feed with E.coli. + Supplementation } \\
\text { of DME @ } 250 \mathrm{~g} / \text { ton feed. }\end{array}$ \\
\hline $\mathbf{T}_{9}$ & Challenge study & Infected with Clostridium spp., on day $8^{\text {th }}$. \\
\hline$T_{10}$ & Prophylaxis & $\begin{array}{l}\text { Infected with Clostridium spp., on day } 8^{\text {th }}+ \\
\text { Supplementation of DME @ } 250 \mathrm{~g} / \text { ton feed. }\end{array}$ \\
\hline $\mathbf{T}_{11}$ & Challenge study & Infected with E.coli on day $8^{\text {th }}$. \\
\hline $\mathbf{T}_{12}$ & Prophylaxis & $\begin{array}{l}\text { Infected with E.coli on day } 8^{\text {th }}+\text { Supplementation of DME } \\
\text { @ } 250 \mathrm{~g} / \text { ton feed. }\end{array}$ \\
\hline $\mathbf{T}_{13}$ & Challenge study* & $\begin{array}{l}\text { Mycotoxin contaminated feed inoculated with Clostridium } \\
\text { spp., and E.coli from day } 8^{\text {th }} \text {. }\end{array}$ \\
\hline $\mathbf{T}_{14}$ & Prophylaxis* & $\begin{array}{l}\text { Mycotoxin contaminated feed inoculated with Clostridium } \\
\text { spp., and E.coli from day } 8^{\text {th }}+\text { Supplementation of DME @ } \\
250 \mathrm{~g} / \text { ton feed. }\end{array}$ \\
\hline
\end{tabular}

* Naturally Aflatoxin contaminated feed in the field level @ 50 ppb

Each bird in treatment group 9,10,13 and 14 were infected orally with approx. $0.4 \mathrm{ml}$ inoculum (bacterial count $1 \times 10^{6}$ CFU/ml) containing Clostridium spp., on $8^{\text {th }}$ day of age. Each bird in group 11, 12, 13 and 14 were infected orally with approx. $0.4 \mathrm{ml}$ inoculum (bacterial count $1 \times 10^{6} \mathrm{CFU} / \mathrm{ml}$ ) containing E.coli on $8^{\text {th }}$ day of age. 
Table.2 Ingredient (\%) composition and proximate composition of the broiler pre-starter, starter and finisher rations

\begin{tabular}{|l|c|c|c|}
\hline \multicolumn{1}{|c|}{ Feed Ingredients (\%) } & Pre-starter & Starter & Finisher \\
\hline Maize & 55.10 & 56.30 & 61.80 \\
\hline Soyabean meal & 39.50 & 37.20 & 30.70 \\
\hline Salt & 0.30 & 0.30 & 0.30 \\
\hline Calcite & 1.70 & 1.70 & 1.60 \\
\hline Di-Calcium Phosphate (DCP) & 1.00 & 0.90 & 0.90 \\
\hline Rice bran oil & 1.80 & 3.10 & 4.20 \\
\hline & Additives (\%) & & \\
\hline NSP degrading enzyme & 0.050 & 0.050 & 0.050 \\
\hline Phytase-2500 IU & 0.02 & 0.02 & 0.02 \\
\hline DL-Methionine & 0.27 & 0.27 & 0.25 \\
\hline Lysine & 0.16 & 0.16 & 0.18 \\
\hline Threonine & 0.02 & 0.02 & 0.03 \\
\hline Sodium bicarbonate & 0.14 & 0.07 & 0.05 \\
\hline Broiler mineral premix (Trouw) & 0.20 & 0.20 & 0.20 \\
\hline Broiler vitamin premix & 0.10 & 0.10 & 0.10 \\
\hline Salinomycin & 0.05 & 0.05 & 0.05 \\
\hline Anti-oxidant (Endoxdry) & 0.01 & 0.01 & 0.01 \\
\hline Vitamin E (50 \%) & 0.010 & 0.008 & 0.005 \\
\hline Lysoforte & 0.05 & 0.05 & 0.05 \\
\hline Choline chloride (60\%) & 0.10 & 0.10 & 0.10 \\
\hline Hepatocare (Liver protectants) & 0.10 & 0.10 & 0.10 \\
\hline Grand Total & 100 & 100 & 100 \\
\hline Pry matter (\%) & 91.30 & 90.93 & 91.74 \\
\hline Crude protein (\%) & 22.47 & 21.55 & 19.20 \\
\hline Crude fibre (\%) & 4.62 & 3.34 & 3.09 \\
\hline Ether extract (\%) & 7.96 & 5.67 & 6.89 \\
\hline Total ash (\%) & 53.07 & 52.52 & 55.07 \\
\hline Nitrogen free extract (\%) & 3,000 & 3,100 & 3,200 \\
\hline $\begin{array}{l}\text { Metabolisable energy } \\
\text { calculated }\end{array}$ & & & \\
\hline & composit/kg) & & \\
\hline & & & \\
\hline
\end{tabular}


Int.J.Curr.Microbiol.App.Sci (2019) 8(7): 2334-2342

Table.3 Serum mineral profile of broilers fed with DME during starter $\left(28^{\text {th }}\right.$ day) and finisher $\left(42^{\text {nd }}\right.$ day) phase

\begin{tabular}{|c|c|c|c|c|c|c|c|c|}
\hline \multirow[t]{2}{*}{$\begin{array}{c}\text { Treatmen } \\
\mathbf{t}\end{array}$} & \multicolumn{2}{|c|}{ Sodium $(\mathbf{m E q} / \mathbf{L})$} & \multicolumn{2}{|c|}{$\begin{array}{l}\text { Potassium } \\
(\mathrm{mEq} / \mathrm{L})\end{array}$} & \multicolumn{2}{|c|}{ Calcium (mEq/L) } & \multicolumn{2}{|c|}{ Chloride $(\mathrm{mEq} / \mathrm{L})$} \\
\hline & $28^{\text {th }}$ day & $42^{\text {nd }}$ day & $\begin{array}{l}28^{\text {th }} \\
\text { day }\end{array}$ & $42^{\text {nd }}$ day & $\begin{array}{l}28^{\text {th }} \\
\text { day }\end{array}$ & $\begin{array}{l}42^{\text {nd }} \\
\text { day }\end{array}$ & $28^{\text {th }}$ day & $\begin{array}{l}42^{\text {nd }} \\
\text { day }\end{array}$ \\
\hline $\mathbf{T}_{1}$ & $\begin{array}{l}158.67 \\
\pm 9.64^{\mathrm{e}}\end{array}$ & $\begin{array}{l}158.19 \\
\pm 3.50^{\text {cd }}\end{array}$ & $\begin{array}{r}13.75 \\
\pm 8.55\end{array}$ & $\begin{array}{c}11.34 \\
\pm 1.96^{\mathrm{a}}\end{array}$ & $\begin{array}{c}9.12 \\
\pm 0.87^{\mathrm{ab}}\end{array}$ & $\begin{array}{c}9.50 \\
\pm 0.75\end{array}$ & $\begin{array}{l}904.54 \\
\pm 23.63\end{array}$ & $\begin{array}{r}789.09 \\
\pm 23.63\end{array}$ \\
\hline $\mathbf{T}_{2}$ & $\begin{array}{l}159.64 \\
\pm 5.91^{\mathrm{e}}\end{array}$ & $\begin{array}{c}140.59 \\
\pm 2.17^{\text {abcd }}\end{array}$ & $\begin{array}{r}11.01 \\
\pm 3.98\end{array}$ & $\begin{array}{c}15.38 \\
\pm 6.53^{\mathrm{ab}}\end{array}$ & $\begin{array}{c}9.31 \\
\pm 0.81^{\mathrm{a}}\end{array}$ & $\begin{array}{c}9.68 \\
\pm 0.31\end{array}$ & $\begin{array}{r}900.00 \\
\pm 70.90\end{array}$ & $\begin{array}{r}832.72 \\
\pm 19.09\end{array}$ \\
\hline $\mathbf{T}_{\mathbf{3}}$ & $\begin{array}{l}108.27 \\
\pm 3.13^{\mathrm{b}}\end{array}$ & $\begin{array}{l}164.71 \\
\pm 6.99^{d}\end{array}$ & $\begin{array}{c}8.34 \\
\pm 2.42\end{array}$ & $\begin{array}{c}25.76 \\
\pm 2.69^{\mathrm{b}}\end{array}$ & $\begin{array}{c}8.43 \\
\pm 0.93^{\mathrm{a}}\end{array}$ & $\begin{array}{c}8.56 \\
\pm 0.18\end{array}$ & $\begin{array}{c}862.72 \\
\pm 15.45\end{array}$ & $\begin{array}{r}834.54 \\
\pm 21.81\end{array}$ \\
\hline $\mathbf{T}_{4}$ & $\begin{array}{c}142.28 \\
\pm 9.16^{\text {bcde }}\end{array}$ & $\begin{array}{r}129.50 \\
\pm 4.09^{\mathrm{ab}}\end{array}$ & $\begin{array}{r}11.80 \\
\pm 6.26\end{array}$ & $\begin{array}{c}13.07 \\
\pm 4.23^{\mathrm{ab}}\end{array}$ & $\begin{array}{c}9.25 \\
\pm 0.37^{\mathrm{a}}\end{array}$ & $\begin{array}{c}9.50 \\
\pm 0.87\end{array}$ & $\begin{array}{r}857.27 \\
\pm 33.63\end{array}$ & $\begin{array}{r}737.27 \\
\pm 77.27\end{array}$ \\
\hline $\mathbf{T}_{5}$ & $\begin{array}{c}62.70 \\
\pm 2.89^{\mathrm{a}}\end{array}$ & $\begin{array}{c}154.34 \\
\pm 2.41^{\text {bcd }}\end{array}$ & $\begin{array}{c}7.84 \\
\pm 2.92\end{array}$ & $\begin{array}{c}20.76 \\
\pm 9.23^{\mathrm{ab}}\end{array}$ & $\begin{array}{c}9.25 \\
\pm 0.37^{\mathrm{a}}\end{array}$ & $\begin{array}{c}9.56 \\
\pm 0.68\end{array}$ & $\begin{array}{r}870.00 \\
\pm 20.90\end{array}$ & $\begin{array}{r}865.45 \\
\pm 13.63\end{array}$ \\
\hline $\mathbf{T}_{6}$ & $\begin{array}{c}124.19 \\
\pm 2.98^{\text {bcde }}\end{array}$ & $\begin{array}{l}124.19 \\
\pm 2.17^{\mathrm{a}}\end{array}$ & $\begin{array}{c}8.26 \\
\pm 2.11\end{array}$ & $\begin{array}{c}16.53 \\
\pm 3.46^{\mathrm{ab}}\end{array}$ & $\begin{array}{r}9.50 \\
\pm 0.25^{\mathrm{ab}}\end{array}$ & $\begin{array}{c}9.87 \\
\pm 0.75\end{array}$ & $\begin{array}{c}872.72 \\
\pm 16.36\end{array}$ & $\begin{array}{r}792.72 \\
\pm 23.63\end{array}$ \\
\hline $\mathbf{T}_{7}$ & $\begin{array}{c}50.40 \\
\pm 1.68^{\mathrm{a}}\end{array}$ & $\begin{array}{l}121.78 \\
\pm 4.09^{\mathrm{a}}\end{array}$ & $\begin{array}{c}5.75 \\
\pm 1.17\end{array}$ & $\begin{array}{c}16.34 \\
\pm 1.57^{\mathrm{ab}}\end{array}$ & $\begin{array}{l}9.31 \pm \\
0.56^{\mathrm{a}}\end{array}$ & $\begin{array}{c}9.62 \\
\pm 0.37\end{array}$ & $\begin{array}{c}831.81 \\
\pm 59.09\end{array}$ & $\begin{array}{r}791.81 \\
\pm 30.00\end{array}$ \\
\hline $\mathbf{T}_{8}$ & $\begin{array}{c}115.99 \\
\pm 8.92^{\mathrm{bc}}\end{array}$ & $\begin{array}{c}154.82 \\
\pm 3.98^{\text {bcd }}\end{array}$ & $\begin{array}{r}11.92 \\
\pm 4.23\end{array}$ & $\begin{array}{c}24.80 \\
\pm 2.50^{\mathrm{b}}\end{array}$ & $\begin{array}{c}9.25 \\
\pm 0.12^{\mathrm{a}}\end{array}$ & $\begin{array}{c}9.37 \\
\pm 0.87\end{array}$ & $\begin{array}{r}895.45 \\
\pm 71.81\end{array}$ & $\begin{array}{r}793.63 \\
\pm 44.54\end{array}$ \\
\hline $\mathbf{T}_{9}$ & $\begin{array}{c}154.09 \\
\pm 0.24^{\mathrm{de}}\end{array}$ & $\begin{array}{c}151.92 \\
\pm 4.82^{\text {bcd }}\end{array}$ & $\begin{array}{c}6.94 \\
\pm 1.51\end{array}$ & $\begin{array}{c}8.26 \\
\pm 1.34^{\mathrm{a}}\end{array}$ & $\begin{array}{c}9.25 \\
\pm 0.37^{\mathrm{a}}\end{array}$ & $\begin{array}{c}9.00 \\
\pm 0.75\end{array}$ & $\begin{array}{c}859.09 \\
\pm 50.00\end{array}$ & $\begin{array}{r}798.18 \\
\pm 23.63\end{array}$ \\
\hline$T_{10}$ & $\begin{array}{l}109.48 \\
\pm 8.32^{\mathrm{b}}\end{array}$ & $\begin{array}{c}134.56 \\
\pm 4.82^{\mathrm{abc}}\end{array}$ & $\begin{array}{c}9.42 \\
\pm 3.65\end{array}$ & $\begin{array}{c}14.23 \\
\pm 1.15^{\mathrm{ab}}\end{array}$ & $\begin{array}{c}9.68 \\
\pm 0.43^{\mathrm{ab}}\end{array}$ & $\begin{array}{c}9.00 \\
\pm 0.37\end{array}$ & $\begin{array}{r}860.00 \\
\pm 32.72\end{array}$ & $\begin{array}{r}796.36 \\
\pm 45.45\end{array}$ \\
\hline$T_{11}$ & $\begin{array}{c}148.07 \\
\pm 2.96^{\text {cde }}\end{array}$ & $\begin{array}{c}130.94 \\
\pm 8.44^{\mathrm{ab}}\end{array}$ & $\begin{array}{r}4.90 \\
\pm 1.55\end{array}$ & $\begin{array}{c}11.53 \\
\pm 1.38^{\mathrm{a}}\end{array}$ & $\begin{array}{c}9.81 \\
\pm 0.93^{\mathrm{a}}\end{array}$ & $\begin{array}{c}9.87 \\
\pm 0.62\end{array}$ & $\begin{array}{r}849.09 \\
\pm 78.18\end{array}$ & $\begin{array}{r}757.27 \\
\pm 99.09\end{array}$ \\
\hline$T_{12}$ & $\begin{array}{c}111.65 \\
\pm 9.05^{\mathrm{bc}}\end{array}$ & $\begin{array}{c}134.32 \\
\pm 7.95^{\text {abc }}\end{array}$ & $\begin{array}{c}7.76 \\
\pm 2.69\end{array}$ & $\begin{array}{c}10.76 \\
\pm 2.69^{\mathrm{a}}\end{array}$ & $\begin{array}{c}9.37 \\
\pm 0.62^{\mathrm{b}}\end{array}$ & $\begin{array}{c}9.93 \\
\pm 0.93\end{array}$ & $\begin{array}{l}818.18 \\
\pm 10.90\end{array}$ & $\begin{array}{r}826.36 \\
\pm 17.27\end{array}$ \\
\hline $\mathbf{T}_{13}$ & $\begin{array}{c}140.35 \\
\pm 1.92^{\text {bcde }}\end{array}$ & $\begin{array}{c}132.15 \\
\pm 6.27^{\mathrm{abc}}\end{array}$ & $\begin{array}{c}6.05 \\
\pm 1.28\end{array}$ & $\begin{array}{c}9.23 \\
\pm 1.53^{\mathrm{a}}\end{array}$ & $\begin{array}{c}9.13 \\
\pm 0.98^{\mathrm{ab}}\end{array}$ & $\begin{array}{c}9.06 \\
\pm 0.06\end{array}$ & $\begin{array}{r}794.54 \\
\pm 15.45\end{array}$ & $\begin{array}{r}862.72 \\
\pm 13.63\end{array}$ \\
\hline$T_{14}$ & $\begin{array}{c}117.20 \\
\pm 3.37^{\mathrm{bcd}}\end{array}$ & $\begin{array}{c}145.90 \\
\pm 3.36^{\text {abcd }}\end{array}$ & $\begin{array}{c}20.19 \\
\pm 9.80\end{array}$ & $\begin{array}{c}10.38 \\
\pm 2.69^{\mathrm{a}}\end{array}$ & $\begin{array}{r}9.43 \\
\pm 0.31^{\mathrm{ab}}\end{array}$ & $\begin{array}{c}9.74 \\
\pm 0.37\end{array}$ & $\begin{array}{l}797.27 \\
\pm 48.18\end{array}$ & $\begin{array}{r}824.54 \\
\pm 50.00\end{array}$ \\
\hline
\end{tabular}

Means with different superscript within the same column differ significantly $(*-\mathrm{P}<0.05$ ) 
Table.4 Serum biochemical parameters of broilers fed with DME during starter $\left(28^{\text {th }}\right.$ day) and finisher $\left(42^{\text {nd }}\right.$ day) phase

\begin{tabular}{|c|c|c|c|c|c|c|}
\hline \multirow{2}{*}{ Treatment } & \multicolumn{2}{|c|}{ Glucose (mg/dl) } & \multicolumn{2}{c|}{ Protein $(\mathbf{g \%})$} & \multicolumn{2}{c|}{ Uric Acid (mcg/dl) } \\
\cline { 2 - 7 } & $\mathbf{2 8}^{\text {th }}$ day & $\mathbf{4 2}^{\text {nd }} \mathbf{d a y}$ & $\mathbf{2 8}^{\text {th }}$ day & $\mathbf{4 2}^{\text {nd }}$ day & $\mathbf{2 8}^{\text {th }}$ day & $\mathbf{4 2}^{\text {nd }}$ day \\
\hline $\mathbf{T}_{\mathbf{1}}$ & $277.17 \pm 6.30$ & $275.00 \pm 4.13$ & $4.94 \pm 0.45$ & $4.39 \pm 0.13$ & $501^{\mathrm{a}} \pm 2.66$ & $603^{\mathrm{a}} \pm 2.28$ \\
\hline $\mathbf{T}_{\mathbf{2}}$ & $285.86 \pm 8.08$ & $278.47 \pm 9.78$ & $4.10 \pm 0.68$ & $4.39 \pm 0.19$ & $507^{\mathrm{a}} \pm 3.80$ & $617^{\mathrm{a}} \pm 0.05$ \\
\hline $\mathbf{T}_{\mathbf{3}}$ & $280.43 \pm 3.04$ & $288.04 \pm 7.60$ & $4.67 \pm 0.43$ & $4.43 \pm 0.78$ & $553^{\mathrm{c}} \pm 6.90$ & $651^{\mathrm{b}} \pm 13.91$ \\
\hline $\mathbf{T}_{\mathbf{4}}$ & $283.69 \pm 6.13$ & $274.34 \pm 2.73$ & $4.28 \pm 0.55$ & $4.55 \pm 0.08$ & $511^{\mathrm{a}} \pm 14.34$ & $621^{\mathrm{a}} \pm 12.71$ \\
\hline $\mathbf{T}_{\mathbf{5}}$ & $299.13 \pm 7.82$ & $284.13 \pm 5.43$ & $4.10 \pm 0.24$ & $4.66 \pm 0.28$ & $501^{\mathrm{a}} \pm 5.43$ & $606^{\mathrm{a}} \pm 0.10$ \\
\hline $\mathbf{T}_{\mathbf{6}}$ & $279.78 \pm 2.82$ & $288.47 \pm 4.21$ & $4.11 \pm 0.74$ & $4.71 \pm 0.16$ & $515^{\mathrm{a}} \pm 8.47$ & $611^{\mathrm{a}} \pm 0.86$ \\
\hline $\mathbf{T}_{\mathbf{7}}$ & $291.52 \pm 6.30$ & $280.65 \pm 3.26$ & $4.11 \pm 1.14$ & $4.96 \pm 0.69$ & $565^{\mathrm{c}} \pm 2.66$ & $632^{\mathrm{ab}} \pm 7.17$ \\
\hline $\mathbf{T}_{\mathbf{8}}$ & $286.95 \pm 8.69$ & $282.39 \pm 4.13$ & $4.63 \pm 0.80$ & $4.26 \pm 0.37$ & $503^{\mathrm{a}} \pm 0.32$ & $619^{\mathrm{a}} \pm 5.00$ \\
\hline $\mathbf{T}_{\mathbf{9}}$ & $276.08 \pm 6.52$ & $271.73 \pm 3.13$ & $4.63 \pm 0.09$ & $4.41 \pm 0.24$ & $508^{\mathrm{a}} \pm 2.01$ & $637^{\mathrm{ab}} \pm 12.55$ \\
\hline $\mathbf{T}_{\mathbf{1 0}}$ & $299.34 \pm 7.34$ & $282.39 \pm 3.26$ & $4.36 \pm 0.16$ & $4.57 \pm 0.26$ & $518^{\mathrm{ab}} \pm 16.63$ & $622^{\mathrm{a}} \pm 4.56$ \\
\hline $\mathbf{T}_{\mathbf{1 1}}$ & $285.86 \pm 7.82$ & $273.04 \pm 5.34$ & $4.88 \pm 0.28$ & $4.54 \pm 0.45$ & $522^{\mathrm{a}} \pm 3.75$ & $609^{\mathrm{a}} \pm 1.73$ \\
\hline $\mathbf{T}_{\mathbf{1 2}}$ & $287.39 \pm 3.91$ & $293.47 \pm 9.00$ & $4.30 \pm 0.44$ & $4.94 \pm 0.01$ & $501^{\mathrm{a}} \pm 0.16$ & $614^{\mathrm{a}} \pm 11.63$ \\
\hline $\mathbf{T}_{\mathbf{1 3}}$ & $282.60 \pm 7.39$ & $286.52 \pm 2.73$ & $4.00 \pm 1.07$ & $4.95 \pm 0.68$ & $545^{\mathrm{bc}} \pm 0.59$ & $654^{\mathrm{c}} \pm 6.90$ \\
\hline $\mathbf{T}_{\mathbf{1 4}}$ & $289.52 \pm 5.26$ & $273.91 \pm 4.34$ & $4.97 \pm 0.55$ & $5.02 \pm 0.09$ & $520^{\mathrm{ab}} \pm 1.08$ & $629^{\mathrm{a}} \pm 8.31$ \\
\hline
\end{tabular}

Means with different superscript within the same column differ significantly $(*$ - $\mathrm{P}<0.05$ )

Table.5 Serum enzyme levels of broilers fed with DME during starter ( $28^{\text {th }}$ day) and Finisher $\left(42^{\text {nd }}\right.$ day) phase

\begin{tabular}{|c|c|c|c|c|c|c|}
\hline \multirow{2}{*}{ Treatment } & \multicolumn{2}{|c}{ SGOT (U/L) } & \multicolumn{2}{c|}{ SGPT (U/L) } & \multicolumn{2}{c|}{ ALP (U/L) } \\
\cline { 2 - 6 } & $\mathbf{2 8}^{\text {th }} \mathbf{d a y}$ & $\mathbf{4 2}^{\text {nd }} \mathbf{d a y}$ & $\mathbf{2 8}^{\text {th }} \mathbf{d a y}$ & $\mathbf{4 2}^{\text {nd }} \mathbf{d a y}$ & $\mathbf{2 8}^{\text {th }}$ day & $\mathbf{4 2}^{\text {nd }}$ day \\
\hline $\mathbf{T}_{\mathbf{1}}$ & $513 \pm 14$ & $649 \pm 16$ & $424 \pm 08$ & $561 \pm 19$ & $848 \pm 17$ & $930 \pm 22$ \\
\hline $\mathbf{T}_{\mathbf{2}}$ & $567 \pm 15$ & $680 \pm 18$ & $459 \pm 06$ & $566 \pm 17$ & $843 \pm 14$ & $980 \pm 24$ \\
\hline $\mathbf{T}_{\mathbf{3}}$ & $570 \pm 13$ & $676 \pm 19$ & $462 \pm 07$ & $557 \pm 18$ & $868 \pm 16$ & $1003 \pm 27$ \\
\hline $\mathbf{T}_{\mathbf{4}}$ & $500 \pm 11$ & $686 \pm 21$ & $483 \pm 09$ & $550 \pm 16$ & $833 \pm 21$ & $981 \pm 21$ \\
\hline $\mathbf{T}_{\mathbf{5}}$ & $567 \pm 11$ & $647 \pm 17$ & $471 \pm 03$ & $533 \pm 13$ & $825 \pm 25$ & $992 \pm 19$ \\
\hline $\mathbf{T}_{\mathbf{6}}$ & $561 \pm 09$ & $622 \pm 14$ & $473 \pm 04$ & $518 \pm 19$ & $803 \pm 18$ & $980 \pm 20$ \\
\hline $\mathbf{T}_{\mathbf{7}}$ & $508 \pm 07$ & $614 \pm 12$ & $478 \pm 09$ & $589 \pm 12$ & $876 \pm 16$ & $944 \pm 18$ \\
\hline $\mathbf{T}_{\mathbf{8}}$ & $554 \pm 12$ & $685 \pm 16$ & $404 \pm 08$ & $558 \pm 17$ & $851 \pm 14$ & $921 \pm 16$ \\
\hline $\mathbf{T}_{\mathbf{9}}$ & $517 \pm 08$ & $616 \pm 17$ & $405 \pm 06$ & $598 \pm 16$ & $863 \pm 17$ & $972 \pm 21$ \\
\hline $\mathbf{T}_{\mathbf{1 0}}$ & $515 \pm 11$ & $699 \pm 14$ & $449 \pm 07$ & $502 \pm 18$ & $855 \pm 12$ & $960 \pm 23$ \\
\hline $\mathbf{T}_{\mathbf{1 1}}$ & $534 \pm 15$ & $622 \pm 11$ & $458 \pm 09$ & $558 \pm 13$ & $830 \pm 16$ & $994 \pm 28$ \\
\hline $\mathbf{T}_{\mathbf{1 2}}$ & $528 \pm 11$ & $681 \pm 15$ & $441 \pm 08$ & $589 \pm 15$ & $812 \pm 15$ & $989 \pm 19$ \\
\hline $\mathbf{T}_{\mathbf{1 3}}$ & $578 \pm 09$ & $644 \pm 10$ & $486 \pm 10$ & $515 \pm 18$ & $801 \pm 17$ & $999 \pm 16$ \\
\hline $\mathbf{T}_{\mathbf{1 4}}$ & $530 \pm 06$ & $610 \pm 16$ & $466 \pm 09$ & $516 \pm 21$ & $831 \pm 21$ & $991 \pm 17$ \\
\hline
\end{tabular}

Means with different superscript within the same column differ significantly $(*-\mathrm{P}<0.05)$ 
Both liver and kidney are critical in detoxification and excretion of mycotoxin contamination in animals hence, their morphological changes leading to high concentrations of the hepatic and nephritic enzymes in the blood (Ademola et al., 2015). However, in present study there was no increase in GOT, AST and ALP during the starter and finisher phase, it could be concluded that supplementation of DME did not cause any liver damage or other disorders where from these enzymes secreted. Detoxifying enzyme is an alternative to the use of live microbes to neutralize mycotoxins in animal feed. It is the application of enzymes responsible for the degradation of mycotoxins (Devreese et al., 2012). So it could be inferred that the supplementation of DME did not cause any adverse health effect on broilers.

\section{Acknowledgement}

The authors are grateful to the Dean, Veterinary College and Research Institute, Namakkal and Tamil Nadu veterinary Animal Sciences University, Chennai, Tamil Nadu for providing necessary facilities to carry out this work.

\section{References}

Ademola S. G., Shittu M. D., Osanyande M. O. and Omidiji, O. E. 2015. Effects of mycotoxin detoxifier supplementation to contaminated groundnut cake on the growth performance, organs and serum enzymes of two broiler strains. Int. J Agri. Inno. Res. 4(2):253-257.

AOAC. 2002. Official Method of Analysis $17^{\text {th }}$ Edn., Washington, DC.

Bagherzadeh Kasmani, F., Karimi Torshizi, M.A., Allameh, A. and Shariatmadari, F. A. 2012. Novel aflatoxin-binding Bacillus probiotic: Performance, serum biochemistry, and immunological parameters in Japanese quail. Poult. Sci. 91: 1846-1853.

Darwish, H.R., Omara, E.A., Abdel, K.B.B., Nada, S.A. and Tawfek, N.S. 2011. Sacchromyces cerevisiae aflatoxininduced toxicity in male albino mice. Report and opinion. 3:32-43.

Devreese, M., Osselaere, A., Goossens, J., Vandenbroucke, V., De Baere, S., Eeckhout, M., De Backer, P. and S. Croubels. 2012. New bolus models for in vivo efficacy testing of mycotoxin detoxifying agents in relation to EFSA guidelines assessed using deoxynivalenol in broiler chickens. Food addit. Contam. A. 29: 1101- 1107.

Do, J.H. and Choi, D.K. 2007. Aflatoxins: Detection, toxicity, and biosynthesis. Biotechnol. Bioprocess Eng. 12: 585593.

Dowling, T.S. 1997. Fumonisins and its toxic effects. Cereal Foods World. 42, 13-15.

El-Katcha, M.I., Soltan, M.A., El-Shobokshy, S.A. and Ahmed Shokry. 2017. Protective effect of chemical and biologicalmycotoxin binder on growth performance, serum biochemistry and carcasss traits in broiler chicks fed on aflatoxin contaminated diet. Alex. J. Vet. Sci. 55(1):180-197.

Fan, Y., Zhao, L., Ma, Q., Li, X., Shi, H., Zhou, T., Zhang, J. and Ji, C. 2013. Effects of Bacillus subtilis ANSB060 on growth performance, meat quality and aflatoxin residues in broilers fed moldy peanut meal naturally contaminated with aflatoxins. Food Chem. Toxicol. 59: 748-753.

Food and Agriculture Organization of the United Nations (FAO), 2001. Manual on the Application of the HACCP System in Mycotoxin Prevention and Control; FAO: Rome, Italy.

Guan, S., Ji, C., Zhou, T., Li, J., Ma, Q. and Niu, T. 2008. Aflatoxin B1 degradation by Stenotrophomonas maltophilia and 
other microbes selected using coumarin medium. Int. J. Mol. Sci. 9:1489-1503. Nyirenda, H.E.C. and Makhambera, T.P.E. 1986. The effect of proteinsource on the performance of broilers. Proceedings of a workshop held at Ryall's Hotel, Blantyre, Malawi titled Utilization of agricultural by-products as livestock feeds in Africa by African Research Network for Agricultural Byproducts (ARNAB) edited by O.A. Little and A.N. Said www.fao.org/wairdocs/ilri/x5494e/x549 4e0m.htm
Snedecor C.W., W.G. Cochran. 1994. Statistical methods 6th edn. Iowa State Univ. Press Anes, USA.

Verma, R. and Chakraborty, D. 2008. Emblica officinalis ameliorates ochratoxin-induced lipid peroxidation in the testis of mice. Acta Pol. Pharma. 65: 187

Yunus, A.W., Razzazi-Fazeli, E. and Bohm, J. 2011. Aflatoxin B1 in affecting broiler's performance, immunity, and gastrointestinal tract: a review of history and contemporary issues. Toxins. 3: 566-590.

\section{How to cite this article:}

Senthilkumar, S., R. Kavitha, S.R. Janani, K.P. Prabhakaran, P. Vasanthakumar, M.R. Purushothaman and Chandrasekaran, D. 2019. Serum Biochemistry of Broiler Chicken Supplemented with Detoxifying Microbial Enzymes to Ameliorate Feed Toxins. Int.J.Curr.Microbiol.App.Sci. 8(07): 2334-2342. doi: https://doi.org/10.20546/ijcmas.2019.807.286 